\title{
Carcinogen Metabolism
}

National Cancer Institute

\section{Source}

National Cancer Institute. Carcinogen Metabolism. NCI Thesaurus. Code C19390.

The sum of the chemical and physical changes occurring in tissue and resulting in biodegradation of any cancer-producing substance or organism. 